\title{
Treat-to-target in systemic lupus erythematosus: recommendations from an international task force
}

\author{
Ronald F van Vollenhoven, ${ }^{1}$ Marta Mosca, ${ }^{2}$ George Bertsias, ${ }^{3}$ David Isenberg, ${ }^{4}$ \\ Annegret Kuhn, ${ }^{5}$ Kirsten Lerstrøm, ${ }^{6}$ Martin Aringer, ${ }^{7}$ Hendrika Bootsma, ${ }_{1}^{8}$ \\ Dimitrios Boumpas, ${ }^{9}$ Ian N Bruce, ${ }^{10}$ Ricard Cervera, ${ }^{11}$ Ann Clarke, ${ }^{12}$ \\ Nathalie Costedoat-Chalumeau, ${ }^{13}$ László Cziriák, ${ }_{1}^{14}$ Ronald Derksen, ${ }^{15}$ \\ Thomas Dörner, ${ }^{16}$ Caroline Gordon, ${ }_{1}^{17}$ Winfried Graninger, ${ }^{18}$ Frédéric Houssiau, ${ }^{19}$ \\ Murat Inanc, ${ }^{20}$ Søren Jacobsen, ${ }_{1}^{21}$ David Jayne, ${ }^{22}$ Anna Jedryka-Goral, ${ }_{1}^{23}$ \\ Adrian Levitsky, ${ }^{1}$ Roger Levy, ${ }^{24}$ Xavier Mariette, ${ }^{25}$ Eric Morand, ${ }^{26}$ Sandra Navarra, ${ }^{27}$ \\ Irmgard Neumann, ${ }^{28}$ Anisur Rahman, ${ }^{29}$ Jozef Rovenský, ${ }^{30}$ Josef Smolen, ${ }^{31}$ \\ Carlos Vasconcelos, ${ }^{32}$ Alexandre Voskuyl ${ }^{33}$ Anne Voss, ${ }^{34}$ Helena Zakharova, ${ }^{35}$ \\ Asad Zoma, ${ }^{36}$ Matthias Schneider ${ }^{37}$
}

\begin{abstract}
Handling editor Tore K Kvien
- Additional material is published online only. To view please visit the journal online (http://dx.doi.org/10.1136/ annrheumdis-2013-205139).

For numbered affiliations see end of article.

\section{Correspondence to} Dr Ronald van Vollenhoven, Department of Medicine, Unit for Clinical Therapy Research, Inflammatory Diseases, Stockholm, Sweden, Karolinska Institutet

Stockholm 17176, Sweden; ronald.van.vollenhoven@ki.se
\end{abstract}

Received 23 December 2013 Revised 22 March 2014 Accepted 23 March 2014

\section{To cite: van}

Vollenhoven RF, Mosca M, Bertsias G, et al. Ann Rheum Dis 2014;73: 958-967.

\section{ABSTRACT}

The principle of treating-to-target has been successfully applied to many diseases outside rheumatology and more recently to rheumatoid arthritis. Identifying appropriate therapeutic targets and pursuing these systematically has led to improved care for patients with these diseases and useful guidance for healthcare providers and administrators. Thus, an initiative to evaluate possible therapeutic targets and develop treatto-target guidance was believed to be highly appropriate in the management of systemic lupus erythematosus (SLE) patients as well. Specialists in rheumatology, nephrology, dermatology, internal medicine and clinical immunology, and a patient representative, contributed to this initiative. The majority convened on three occasions in 2012-2013. Twelve topics of critical importance were identified and a systematic literature review was performed. The results were condensed and reformulated as recommendations, discussed, modified and voted upon. The finalised bullet points were analysed for degree of agreement among the task force. The Oxford Centre level of evidence (LoE, corresponding to the research questions) and grade of recommendation (GoR) were determined for each recommendation. The 12 systematic literature searches and their summaries led to 11 recommendations. Prominent features of these recommendations are targeting remission, preventing damage and improving quality of life. LoE and GoR of the recommendations were variable but agreement was $>0.9$ in each case. An extensive research agenda was identified, and four overarching principles were also agreed upon. Treat-to-target-in-SLE (T2T/SLE) recommendations were developed by a large task force of multispecialty experts and a patient representative. It is anticipated that 'treating-to-target' can and will be applicable to the care of patients with SLE.

\section{INTRODUCTION}

Over the past 50 years, the therapeutic strategy for some of the most common chronic diseases has evolved from a symptom-based to a target-based approach, under the influence of evidence that such approaches yield superior outcomes. For example, in the management of hypertension, targeting suitably chosen values for systolic or diastolic blood pressure yields long-term reductions in the risks for cardiovascular diseases. ${ }^{1}$ Likewise, in the management of diabetes, targeting specific values for blood-glucose, measured directly or indirectly by haemoglobin A1c, has resulted in major improvements in long-term prognosis. ${ }^{2}$

In the rheumatic diseases, where symptomatology is often the predominant concern on the patient's part, it is less intuitive to investigate targeted approaches. Nonetheless, in the management of rheumatoid arthritis (RA), several randomised clinical trials provided evidence that such targeted approaches yielded superior outcomes in terms of clinical course, long-term damage and functional status. $^{3-5}$ As a result, treat-to-target recommendations were developed for RA, ${ }^{6}$ prompting further investigations and cautious implementation of such approaches. More recently, treat-to-target recommendations for the spondyloarthropathies were also published. ${ }^{7}$

Systemic lupus erythematosus (SLE) is a chronic disease where treatment is typically long-term or even life-long. The disease engenders significant symptomatologies, which are often the principal drivers of the patient's medical need for interventions, however, not all disease activity is perceptible as characteristic symptoms. SLE is also associated with the progressive accumulation of irreversible organ damage, which has been shown to be a predictor of further damage, additional morbidity and early mortality. ${ }^{8}$ Based on these considerations, it was reasonable to ask the question if the principles of treating-to-target could be applied to the management of SLE as well. An international task force was gathered to investigate this question and formulate recommendations aimed at improving the management of SLE in clinical practice through target-based approaches. The task force directed a systematic literature review (SLR), which served as the evidence base, and developed a set of overarching principles and recommendations for treating-to-target in SLE. 


\section{METHODS}

The process followed for deriving these recommendations was based on the treat-to-target initiative in RA. ${ }^{6}$ The idea for this project originated in informal discussion between some of the authors, most notably RvV, MM, JS and MS. A T2T/SLE working group was formed consisting of a patients' representative and 14 medical specialists with expertise in the clinical investigation and treatment of SLE as well as in the process of developing consensus-based guidance documents (additionally, authors RvV, MM, DI, KL and MS had a more practical role and were internally referred to as the 'steering committee'). Financial support was requested and obtained from two companies (specified under Disclosures), but in both cases this support was unrestricted and intended to cover the actual costs of having the meetings including transportation, housing if needed, and meeting facilities. The sponsors had no role in setting the agenda or selecting participants; no company representatives were present at any of the meetings, and the companies had no role in the writing of the manuscript (including no 'writing support'). The working group convened in May and August 2012 to determine the major topics for which a SLR was required through a nominal process. This process resulted in some consensus on the general direction of recommendations and the development of the first draft of provisional overarching principles. Following these meetings and extensive electronic deliberations, the agenda for the systematic literature search was identified as consisting of 12 distinct topics. During the final months of 2012, this literature search was performed by authors GB and AL with continued interactions with, and support from, authors RvV and MM. The PubMed database was searched using index terms, and all English language human studies were evaluated based on the title, abstract and/or full text. The results of the literature search were graded for the level of evidence (LoE) on a scale of 1-5, and the grade of the ensuing recommendation (GoR) was determined on a scale from A (highest) to $\mathrm{D}$ (lowest). ${ }^{9} 10$

On the basis of the SLR, provisional recommendations were developed and were circulated through the entire working group and extensively discussed electronically and modified accordingly. Subsequently, a larger meeting took place in January of 2013, at which a larger number of international experts were present. This group, the T2T/SLE international task force (all authors), consisted of rheumatologists, nephrologists, dermatologists, internists, a clinical immunologist and a patients' representative; and in addition to the larger numbers of European experts, representation was achieved from most regions of the world including North America, South America, Southeast Asia, and Australia/Oceania. During this meeting, discussions took place in plenary and break-out sessions, and the principles of Delphi technique were applied for achieving consensus. The original 12 topics were slightly reorganised (based on considerable overlap in the relevant literature) yielding 11 recommendations. After extensive discussion, revised statements were drafted and voted upon. Greater than $80 \%$ consensus was achieved for all but two of the revised statements. These two statements were discussed further and additional inquiries and clarifications on points of the literature, as well as details of wording, were requested. The final vote was performed by email following the face-to-face meeting, and yielded $>80 \%$ consensus for both statements. Likewise, extensive electronic communications again took place resulting in small additional clarifications of wording and removal of redundant material.

The finalised overarching principles and recommendations were circulated to the entire task force, and the agreement

with each statement was voted upon by all members, on a scale of $0-10$, the highest value indicating the greatest level of agreement.

\section{RESULTS}

The T2T/SLE working group identified 12 areas of key importance to be investigated by a SLR. These areas are shown in table 1 , along with the number of articles retrieved by each PubMed search. The Overarching Principles and Recommendations achieving consensus from the larger International Task Force are shown in box 1 and are discussed in detail below.

\section{Overarching principles}

The task force considered that the central guiding principles towards the treatment for SLE, anchored deeply in humanistic traditions and the nature of medicine, were not suitable for a literature-based review, but could be codified through broad discussion and consensus. Thus, these principles were not investigated through an examination of published data but were extensively debated, circulated and voted upon.

The management of SLE should be based on shared decisions between the informed patient and her or his physician(s)

A key ingredient of the decision-making process in SLE is the patient's autonomy: (s)he must be central in the process and actively involved. The physician would, in most instances, have to be a specialist experienced in the management of SLE, and should work as part of a team, using an interdisciplinary approach. Each therapeutic decision should be individualised and balanced.

Shared decision making is the conversation that happens between a patient and their health professional to reach a healthcare choice together. This conversation needs patients and professionals to understand what is important to the other person when choosing a treatment (NHS 2012, http://sdm. rightcare.nhs.uk/about/shared-decision-making/).

Table 1 Topics selected for the systematic literature search

Topic

Correlation between disease activity and outcomes (damage accrual, mortality, QoL)

Low disease activity and remission as surrogates of therapeutic success against outcomes

Benefits and harms of treating serologically active but clinically quiescent disease

Validated definitions of flares of disease activity against outcomes

Is sustained remission or prevention of flares an achievable goal?

Correlation between chronic, irreversible organ damage and mortality

Correlation between chronic, irreversible organ damage and QoL

Early vs late, and intensive vs less intensive control of disease

activity

'Induction-maintenance' vs 'induction-only' treatment strategy

Long-term benefits and harms of glucocorticoids regimes in chronic

maintenance treatment

Benefits and harms of anticoagulation regimens in patients with antiphospholipid antibodies

Benefits and harms of adjunct therapies 
- Overarching principle 1: The management of systemic lupus erythematosus (SLE) should be based on shared decisions between the informed patient and her/his physician(s).

- Overarching principle 2: Treatment of SLE should aim at ensuring long-term survival, preventing organ damage, and optimising health-related quality-of-life, by controlling disease activity and minimising comorbidities and drug toxicity.

- Overarching principle 3: The management of SLE requires an understanding of its many aspects and manifestations, which may have to be targeted in a multidisciplinary manner.

- Overarching principle 4: Patients with SLE need regular long-term monitoring and review and/or adjustment of therapy.

Recommendations:

1. The treatment target of SLE should be remission of systemic symptoms and organ manifestations or, where remission cannot be reached, the lowest possible disease activity, measured by a validated lupus activity index and/or by organ-specific markers.

2. Prevention of flares (especially severe flares) is a realistic target in SLE and should be a therapeutic goal.

3. It is not recommended that the treatment in clinically asymptomatic patients be escalated based solely on stable or persistent serological activity.

4. Since damage predicts subsequent damage and death, prevention of damage accrual should be a major therapeutic goal in SLE.

5. Factors negatively influencing health-related quality of life (HRQOL), such as fatigue, pain and depression should be addressed, in addition to control of disease activity and prevention of damage.

6. Early recognition and treatment of renal involvement in lupus patients is strongly recommended.

7. For lupus nephritis, following induction therapy, at least 3 years of immunosuppressive maintenance treatment is recommended to optimise outcomes.

8. Lupus maintenance treatment should aim for the lowest glucocorticoid dosage needed to control disease, and if possible, glucocorticoids should be withdrawn completely.

9. Prevention and treatment of antiphospholipid syndrome (APS)-related morbidity should be a therapeutic goal in SLE; therapeutic recommendations do not differ from those in primary APS.

10. Irrespective of the use of other treatments, serious consideration should be given to the use of antimalarials.

11. Relevant therapies adjunctive to any immunomodulation should be considered to control comorbidity in SLE patients.

Treatment of SLE should aim at ensuring long-term survival, preventing organ damage and optimising health-related quality-of-life, by controlling disease activity and minimising comorbidities and drug toxicity

This principle emphasises that, rather than a single target for treatment, the treatment of SLE entails a range of targets that all must be taken into account. The task force discussed whether a 'hierarchy of targets' could be established. Although some elements were self-evident (eg, survival takes precedence over lowering glucocorticoids), the task force was unable to establish a conclusive recommendation regarding a hierarchy of targets.

The management of SLE requires an understanding of its many aspects and manifestations, which may have to be targeted in a multidisciplinary manner

The task force wished to recognise the diversity of clinical expressions of SLE and the ensuing need for healthcare providers to be cognisant and aware of the multifaceted nature of the disease. Moreover, it is recognised that the nature of SLE itself may make it necessary, in many patients' cases, to have care delivered by more than one type of healthcare provider. This requires several specialist physicians working together in the management of one patient or a constellation of medical and paramedical healthcare providers and non-medical professionals working as a team if required.

Patients with SLE need regular long-term monitoring and review and/or adjustment of therapy

As in some other chronic diseases, therapy traditions exist where only acute needs are addressed at times of a medical crisis or when the patient seeks medical help. For SLE, this is felt to be as inappropriate as it is for diabetes mellitus. SLE can cause serious derangements at different organ levels that may not be perceived by the patient until significant damage (renal failure due to nephritis) or a life-threatening situation have developed (neutropenia, thrombocytopenia). The treatment of SLE may likewise engender such risks (immunosuppressives), in particular, if therapeutic agents are continued longer than really needed, or at too high a dose causing serious long-term consequences (glucocorticoids). For all these reasons, patients with SLE should be monitored regularly and, specifically, their treatment needs to be reviewed and adjusted at reasonable time intervals. ${ }^{11}$ Additionally, treatment of SLE must be tailored to the individual patient.

\section{Recommendations}

The treatment target of SLE should be remission of systemic symptoms and organ manifestations or, where remission cannot be reached, the lowest possible disease activity, measured by a validated lupus activity index and/or by organ-specific markers Epidemiological studies with long-term observation period (beyond 5 years) have demonstrated that SLE disease activity, assessed on a single occasion or longitudinally, correlates positively with adverse patient outcomes, such as accrual of irreversible organ damage (quantified by the Systemic Lupus International Collaborating Clinics (SLICC)/American College of Rheumatology SLICC Damage Index (SDI) ${ }^{12}$ ) and mortality (see online supplementary table S1). These associations have been shown for validated global SLE disease activity indices including the SELENA-SLEDAI (average score $>3.5$ during past visits has a HR 1.7 for damage accrual ${ }^{13}$ ), SLEDAI-2K (HR for mortality 1.15 per 1 unit adjusted mean score ${ }^{14}$ ), SLAM-R (HR for damage 1.15 per 1 unit $^{15}$ ), and BILAG (HR for death 1.15 per 1 unit; HR for new organ damage 1.08 per 1 unit $^{16}$ ). Furthermore, moderate or severe organ-specific lupus activity, especially from the haematological, ${ }^{17}{ }^{18}$ neuropsychiatric ${ }^{19}{ }^{20}$ 
and renal domains, ${ }^{21-23}$ is also linked to damage accrual and/or mortality. In accordance with these findings, data from cohort studies and the extended follow-up of controlled trials suggest that attainment of low disease activity or remission predicts favourable long-term patient outcomes. ${ }^{24} 25$ The evidence is strongest for lupus nephritis, ${ }^{26-28}$ where achievement of complete renal response (remission)—usually defined as stable/ improved renal function with low-grade proteinuria $(<0.5-1 \mathrm{~g} /$ $24 \mathrm{~h}$ ) plus/minus inactive urine sediment-after administration of immunosuppressive treatment is associated with significantly lower risk (likelihood ratio $0.14^{26}$ ) for progression to end-stage renal disease (ESRD).

The task force considered whether to define 'remission' for general or organ-specific SLE. It was recognised that no generally accepted definition exists, and that there is disagreement among experts on whether the most appropriate definition of remission would be one that reflects only clinical disease aspects, one that includes clinical and serological aspects, or one that includes both and also puts limits on the treatments that are allowed when a patient is said to be in remission. An earlier EULAR initiative failed to resolve the issue completely. ${ }^{11}$ Definitively defining remission was felt to be outside the scope of the current T2T/SLE project, but the T2T/SLE task force is planning further work in this area. It was noted that recently the Asia-Pacific Lupus Collaboration developed a preliminary definition for a Lupus Low Disease Activity State. ${ }^{29}$

It was also recognised that the concept of 'organ-specific outcomes' may be applicable only to some, but not to other, SLE manifestations.

This recommendation also implies that at least one validated disease activity measure ${ }^{30}$ should be regularly monitored in every SLE patient along with organ-specific markers.

Prevention of flares (especially severe flares) is a realistic target in SLE, and should be a therapeutic goal

Patients with SLE tend to follow a highly variable course with periods of quiescence alternating with flares of activity. In recent therapeutic trials of general SLE, ${ }^{31-34}$ and depending on the disease activity instrument that is used, $64-74 \%$ of patients may experience some degree of disease exacerbation after initial achievement of low disease activity. Severe SLE flares, which typically mandate the use of moderate-to-high doses of glucocorticoids and/or initiation or intensification of immunosuppressive treatment, are encountered in $17-38 \%$ of patients. ${ }^{31-34}$ Likewise, studies in lupus nephritis have shown that exacerbations of renal disease occur in $38-65 \%$, and severe renal flares in $8-26 \%$ of patients who initially responded to immunosuppressive treatment. ${ }^{26} 27$ 35-38 Accordingly, and in spite of the heterogeneity in definitions and follow-up periods, data from large observational and controlled studies suggest that 10-40\% of SLE patients can achieve long-lasting (beyond 1 year) states of absence of flares and/or disease remission. ${ }^{24} 253233 \quad 3739$

A large number of studies have demonstrated that exacerbations of SLE activity (especially severe exacerbations) may adversely impact on long-term patient outcomes, suggesting that prevention of flares may be an important therapeutic goal in SLE. There is ample evidence that renal disease exacerbations, particularly severe nephritic flares-characterised by significant reduction in glomerular filtration rate, reactivation of urine sediment and increase in proteinuria-after initial response to immunosuppressive treatment, carry an increased risk (HR 13.9 $9^{27}$; likelihood ratio $11.8^{26}$ ) for development of irreversible renal damage or death. Similar associations have been demonstrated for neuropsychiatric ${ }^{40} 41$ and general SLE ${ }^{18} \quad 41-44$ flare-ups.

Thus, the task force felt that the literature supported both elements: that flare prevention should be a therapeutic goal, and that it is a realistic target. Of course 'realistic' does not mean that it can be achieved in every patient, but it was felt that the abovereferenced studies on flare prevention have demonstrated sufficiently clearly that there are interventions that can prevent at least some flares with a reasonable balance to risks/side effects.

It is not recommended that the treatment in clinically asymptomatic patients be escalated based solely on stable or persistent serological activity

In a clinically asymptomatic SLE patient with increasing serological activity (increasing anti-double-stranded (ds) DNA titres usually accompanied by decreasing serum complement concentrations), there is an increased risk for developing flare, including severe flare and flare from the kidneys or other major organs. ${ }^{45-53}$ Closer monitoring is therefore advised. However, the predictive value of these tests is modest (ORs ranging 1.8$3.2^{485253}$ ) and thus, in the individual patient, there may be a notable discordance between serological findings and clinical outcome. Nevertheless, two small controlled studies in clinically stable SLE patients with rising anti-dsDNA titres showed that patients who were randomised to receive glucocorticoids (starting dose $30 \mathrm{mg} /$ day prednisone, then gradually tapered off) developed fewer severe flares compared to those who did not (combined relative risk $0.74 ; 95 \%$ CI 0.60 to 0.91 ). ${ }^{54} 55$ However, this approach carries the risk of overtreating patients with glucocorticoids (number needed to treat to prevent one major flare=5), and therefore, the task force concluded that clinically asymptomatic patients with stable or persistent serological activity should not receive treatment escalation solely on account of the serological findings. This is also supported with results from a large cohort. The investigators followed clinically quiescent SLE patients with prolonged (at least 2 years) serological activity for up to 10 years and found that they accrued less damage compared to other SLE patients. ${ }^{56}{ }^{57}$ In these patients, closer monitoring is advised for prompt identification of clinical flare signs.

Since damage predicts subsequent damage and death, prevention of damage accrual should be a major therapeutic goal in SLE Several cohort studies with large numbers of patients have demonstrated that accrual of irreversible organ damage in SLE is a strong prognostic factor for subsequent damage accrual in the same or other organs (HR 1.30 per 1 unit SDI ${ }^{15}$ ) and also for increased mortality (HR 1.40 per 1 unit SDI ${ }^{58}$ ). These associations have been shown for the total SDI score and for individual SDI domains, especially the renal and neuropsychiatric items. $^{22}$ 59-61 Additionally, these effects are irrespective of whether the damage is accrued early (within the first 1-3 years) or later during the disease course, and whether the damage can be attributed directly to the disease, or is considered a consequence of (long-term) treatment, or a concomitant disorder. Thus, prevention of damage includes control of disease activity, prevention of flares and avoiding drug toxicity. Damage is reliably measured by the SDI.

Factors negatively influencing health-related quality of life (HRQoL), such as fatigue, pain and depression should be addressed in addition to control of disease activity and prevention of damage HRQoL is compromised in SLE patients as a result of the disease itself but frequently also due to the coexistence of 
aggravating factors, especially fatigue, pain and depression (reviewed in ${ }^{62}{ }^{63}$ ). Thus, cross-sectional studies have reported weak inverse associations between HRQoL and measures of SLE activity $^{64-66}$ and damage ${ }^{6465}$ 67-69 (correlation coefficients typically in the range of -0.20 to -0.52 ). Similarly, longitudinal changes in disease activity ${ }^{67} 7071$ and damage ${ }^{71-73}$ correlate weakly with respective changes in HRQoL, and therapeutic trials have shown modest concordance between clinical response to treatment and improvement in HRQoL. ${ }^{74-76}$ Therefore, optimising HRQoL should be considered independently in treatment decisions and disease management. Put in other words, it is recommended that the targets referred to earlier (remission, absence of flares, etc) are pursued, while at the same time also aiming for improvements in the many other factors that could adversely impact on HRQoL. The task force discussed whether HRQoL should be an additional therapeutic target but it was considered that all previously indicated targets contribute to HRQoL so that this is implied. From the patient's point of view, the ultimate goal is to 'survive and to survive well'. This also extends to the importance of ensuring the patients' social functioning including fitness to work.

\section{Early recognition and treatment of renal involvement in SLE} patients is strongly recommended

No controlled trials have compared an 'early' versus 'late' therapeutic strategy against hard outcomes in SLE. Nonetheless, extrapolated evidence from observational and therapeutic studies suggests that patients with active severe SLE manifestations may benefit from early recognition and management of their disease. Evidence is stronger for lupus nephritis, where delay in diagnosis (by kidney biopsy) and initiation of immunosuppressive treatment has been associated with increased risk for renal relapses (RR 1.03 per 1-month delay ${ }^{77}$ ) and ESRD (HR 4.2 for delay $>6$ months). ${ }^{78-83}$ Similarly, timely (within 2 weeks) recognition and management of lupus myelopathy correlates with improved outcome. ${ }^{84} 85$ Although the evidence is lacking, in the opinion of the panelist, the same principle may apply to other severe SLE manifestations. As for the intensity of immunosuppressive treatment, meta-analyses of randomised controlled studies in SLE patients with severe inflammatory renal and CNS involvement have shown that the combination of glucocorticoids with immunosuppressive agents is more efficacious than glucocorticoids alone. ${ }^{86} 87$

For lupus nephritis, following induction therapy, at least 3 years of immunosuppressive maintenance treatment is recommended to optimise outcomes

In lupus nephritis, after initial immunosuppressive (induction) treatment, which aims to induce remission by controlling immunologic activity, a subsequent longer period of less intensive (maintenance) treatment is required to consolidate remission and prevent relapses. ${ }^{27}{ }^{88-90}$ Based on the results of controlled trials and their extended follow-up, ${ }^{21} 3591$ it is recommended that the duration of maintenance treatment in lupus nephritis should be at least 3 years. This is also supported by observational studies showing that early (before 1824 months after achieving response) tapering or discontinuation of immunosuppressive treatment is associated with increased risk (RR 2.6) for renal flares. ${ }^{92} 93$ We note that these studies do show that maintenance therapy leads to better results overall, but they do not completely prove that this is true even for those patients who are in complete remission following induction therapy. We believe this could be studied in various clinical trial datasets, and this specific question is, therefore, on the research agenda. We also emphasise that, as is generally the case, this recommendation must be applied in a flexible manner to take into account the patient's individual characteristics.

Although there is lack of evidence, the principle of inductionmaintenance immunosuppressive treatment is followed by many experts in the management of other severe SLE manifestations as well.

SLE maintenance treatment should aim for the lowest glucocorticoid dosage needed to control disease, and if possible, glucocorticoids should be withdrawn completely

A larger number of cohort studies in SLE have demonstrated a significant dose-related association between exposure to systemic glucocorticoids and damage accrual (HR 1.05 per $1 \mathrm{mg},{ }^{15}$ and HR 1.50 for $>6-12 \mathrm{mg}^{94}$ average daily prednisone dose). Most frequently afflicted are the musculoskeletal, cardiovascular, peripheral vascular, ocular and metabolic domains, and the effects seem to be irrespective of the route or formula of glucocorticoid administration. In children with SLE, high cumulative doses of glucocorticoids may also adversely impact on growth. ${ }^{95}$ The task force debated whether there was a safe lower level of glucocorticoid dosing. While this question has been studied in other diseases where no such safe lower level could be identified, ${ }^{96} 97$ there are no studies specifically addressing this issue in SLE. Nonetheless, based on the available evidence and general pharmacological considerations, the task force supported the main recommendation to aim for the lowest glucocorticoid dosage during maintenance treatment, and the comment to withdraw glucocorticoids completely 'if possible'.

Prevention and treatment of antiphospholipid syndrome-related morbidity should be a therapeutic goal in SLE; therapeutic recommendations do not differ from those in primary antiphospholipid syndrome

Antiphospholipid syndrome (APS) is present in approximately $16 \%$ of SLE patients, it contributes to increased damage accrual and has major medical consequences for the patient and her/his medical needs. ${ }^{98-101}$ Thus, awareness of the increased risk of APS in SLE patients should be high to ensure rapid detection. Although there is no randomised evidence to guide primary thromboprophylaxis in SLE patients with positive antiphospholipid antibodies, two cohort studies have demonstrated reduced risk for first thrombosis in patients who were treated with acetylsalicylic acid and/or hydroxychloroquine. ${ }^{100} 102$ In SLE patients with APS and history of thrombotic events, two randomised controlled trials found comparable efficacy of low-intensity (target INR 2.1-3.0) versus high-intensity (target INR >3.0) anticoagulation in preventing recurrent thrombosis. ${ }^{103} 104$ Conversely, two cohort studies that included patients with moderate/high-risk antiphospholipid profile and/or history of arterial thrombotic events, suggested benefit of the high-intensity regimen. ${ }^{105} 106$

The task force felt that a common misconception might be that APS should be treated differently when occurring in the context of SLE. There is no evidence to suggest this, and this fact by itself prompted the task force to include the 'negative' final statement in this recommendation. Thus, it is recommended that prevention and management of APS-related morbidity in SLE patients should be similar to that in primary APS patients.

Irrespective of the use of other treatments, serious consideration should be given to the use of antimalarials

In the context of SLE, the medication class of 'antimalarials' refers primarily to chloroquine and hydroxychloroquine, with 
the latter being the favoured option, where available. Data primarily from non-randomised studies have suggested favourable effects of antimalarials on various SLE outcomes, ${ }^{107}$ such as a reduction of flares (relative risk 0.43 for major flares), ${ }^{108}$ improvement of skin manifestations, ${ }^{109} 110$ prevention of damage accrual (HR 0.55), ${ }^{111} 112$ and possible reduction in mortality risk (HR 0.14-0.62); ${ }^{113-115}$ accordingly, some task force members felt that this therapeutic class should be considered in all SLE patients unless contraindicated. Others, however, pointed out that, while the literature supports the effectiveness of antimalarials per se, the same studies also make it clear that these agents have modest effect sizes that are subject to possible confounding bias and are not free of risks, and thus, favoured more restrained wording. Irrespective of this, contraindications to these medications should of course, be respected. Hydroxychloroquine was favoured, where available, over chloroquine owing to a better safety profile. The antimalarial mepacrine/quinacrine is used in some countries for cutaneous manifestations in SLE.

Relevant therapies adjunctive to any immunomodulation should be considered to control comorbidity in SLE patients

This recommendation reflects on such therapeutic categories as antihypertensives, lipid-lowering agents, antihyperglycemics, antiplatelet/anticoagulants, immunisations, and bone-protecting agents, all of which are supported by strong evidence in the appropriate clinical setting. In SLE patients, the above mentioned treatments seem to be as safe and efficacious as in the general population, ${ }^{116-120}$ although there are no controlled studies to demonstrate benefit against damage accrual or mortality. By contrast, there is poor evidence to support the use of additional adjunctive therapies or complementary medicines to achieve the key therapeutic targets in the management of SLE.

\section{Agreement among experts}

Agreements with the recommendations were assessed on a $0-10$ scale in a final round of electronic voting, and yielded excellent values ranging from 9.03-9.87 (table 2).

\section{Research agenda}

The SLR and the ensuing deliberations highlighted that several important issues related to the management of SLE remain elusive and will require additional, well-designed studies to resolve. Thus, the task force established a research agenda based on these findings (box 2). It should be noted that it is by no means exhaustive, as it only represents those topics that emerged from the SLR and deliberations. Moreover, only such topics were included as could reasonably be approached through current research efforts.

\section{DISCUSSION}

The international task force on treating-to-target in SLE (T2T/ SLE) has developed a set of Overarching Principles and Recommendations that are expected to be the first step in a longterm process. These bullets are directed at experienced physicians and are not meant to replace clinical judgment, knowledge acquired in appropriate training and continuous medical education, and experience acquired in the real-world care of patients with SLE. Moreover, the treatment targets identified here, or the measures used to ascertain them, may have to be adjusted in accordance with patient preferences, comorbidities or risks.

There may, in some situations, be confusion about the word 'target'. Certainly the word 'target' could be used to indicate a molecule or cell type that can be targeted with a medication or
Table 2 Level of evidence, grade of recommendations, and agreement among experts

\begin{tabular}{|c|c|c|c|}
\hline & $\begin{array}{l}\text { Level of } \\
\text { evidence }\end{array}$ & $\begin{array}{l}\text { Grade of } \\
\text { recommendation }\end{array}$ & $\begin{array}{l}\text { Level of } \\
\text { agreement }\end{array}$ \\
\hline \multicolumn{4}{|c|}{ Overarching Principles } \\
\hline 1 & - & - & 9.48 \\
\hline$\|$ & - & - & 9.90 \\
\hline III & - & - & 9.42 \\
\hline IV & - & - & 9.81 \\
\hline \multicolumn{4}{|c|}{ Recommendations } \\
\hline 1 & $3(\mathrm{SLE}) / 1^{*}(\mathrm{LN})$ & $C(S L E) / A(L N)$ & 9.52 \\
\hline 2 & $2(\mathrm{SLE}) / 1^{*}(\mathrm{LN})$ & $B(S L E) / A(L N)$ & 9.32 \\
\hline 3 & 2 & B & 9.03 \\
\hline 4 & $1^{*}$ & A & 9.71 \\
\hline 5 & 2 & B & 9.03 \\
\hline 6 & 2 & B & 9.87 \\
\hline 7 & 2 & B & 9.13 \\
\hline 8 & 2 & B & 9.58 \\
\hline 9 & $3 \dagger$ & C & 9.52 \\
\hline 10 & 2 & B & 9.35 \\
\hline 11 & $4,5 \ddagger$ & C & 9.55 \\
\hline
\end{tabular}

biologic, but in the context of this article 'target' does, of course, not refer to such drug therapy targets but to the therapeutic goal we set for each individual patient.

For treatment-to-target to be successful, as in the case of hypertension, diabetes, or RA, three principal ingredients need to be in place: it must be possible to identify the appropriate target and to measure whether it has been achieved, and appropriate interventions must be available to (attempt to) achieve the target. In the case of SLE, each of these is, to some extent, lacking. Thus, the identification of therapeutic targets in SLE revealed that the evidence in support of some was variable, and more concentrated efforts to define appropriate targets are still needed. High-level evidence for the long-term effectiveness of a targeted-treatment strategy in SLE is not yet available, and the conduct of large prospective randomised trials of targeted versus standard care of SLE are warranted. This will also bear on the issue of measuring the target, and, as already indicated, further work to define the important target of 'remission' is being undertaken. Other appropriate targets may be defined in future prospective trials, but the relevant analyses in this regard of existing large datasets, such as those available from randomised drug trials in SLE, could provide very important additional support. The task force recommends that at least one validated disease activity measure should be regularly monitored. Examples include the BILAG, SLEDAI, ECLAM and others. As there is no clear evidence for using one of them over the other, for now, clinicians can choose the instrument that best suits their clinical situation. Perhaps the weakest link in T2T/SLE is the availability of therapeutic options that would make it possible, in practice, to aim for certain targets. The therapeutic armamentarium for SLE consists of a relatively small number of agents in the therapeutic classes of glucocorticoids, antimalarials, immunosuppressives and biologics. In the latter category, only one agent (belimumab) is approved for use in SLE, and one (rituximab) is used not infrequently 'off-label' in refractory cases. ${ }^{121}{ }^{122}$ Fortunately, several new agents of 


\section{Box 2 Research agenda for some of the 11}

recommendations

Recommendation 1

- Development of definition(s) of remission in systemic lupus erythematosus (SLE)

- Further studies linking specific disease activity states to long-term outcomes

- Definition of a minimal acceptable disease activity

- Longitudinal studies investigating the long-term outcomes of targeted treatment in non-renal SLE

- Prospective randomised trial comparing targeted versus standard treatment in SLE

Recommendation 2

- Development and/or refinement of flare definitions and flare assessment tools

- Prospective trials, including active-treatment trials as well as withdrawal trials, to assess prevention of flare

Recommendation 4

- A meta-analysis of the numerous observational studies linking damage to mortality

- A study specifically demonstrating that damage prevention leads to gains in health-related quality of life (HRQoL)

Recommendation 5

- Definition of a set of key patient-reported outcomes in SLE

- Studies of non-inflammatory factors influencing HR-QoL in patients with SLE

- Studies of interventions targeting such factors

Recommendation 7

- Prospective studies to investigate early intensive therapy and the principle of induction/maintenance in non-renal lupus

- Studies based on existing clinical trial datasets to determine if the general rule that maintenance therapy leads to better results is true even for those patients who are in complete remission following induction therapy.

Recommendation 8

- Studies to determine if a 'safe' lower level of long-term glucocorticoid exposure can be identified

- Studies on glucocorticoid withdrawal

Recommendation 9

- Therapeutic studies in SLE antiphospholipid syndrome with immunosuppressives/immunomodulators

- Studies on the feasibility of discontinuing anticoagulant therapy following immunomodulation to suppress the production of antiphospholipid antibodies

Recommendation 10

- Studies to determine if hydroxychloroquine must be recommended or not in every patient with lupus. For how long? At what dosage? And with or without monitoring of serum drug level?

Recommendation 11

- More studies on complementary interventions in SLE

considerable interest are in development for the treatment of SLE, raising expectations that it will soon be possible to aim for therapeutic targets with greater confidence that they can be achieved. Thus, we regard this work as a first proactive step towards a future where many new agents for SLE will make it imperative to use them in the best possible manner

Recognising that many female patients with SLE are of childbearing age, the task force considered it important to emphasise that the application of the treat-to-target principles may be particularly challenging in the peripregnancy setting. The use of various classes of medications that would otherwise be considered in the application of treat-to-target to the individual patient might, in some instances, be inappropriate when the wish to conceive raises greater concerns for the well-being of the patient or their offspring.

In summary, treat-to-target-in-SLE (T2T/SLE) recommendations were developed by a large task force of multispecialty experts and a patient representative. The level of the evidence was variable, but overall sufficient to provide acceptable strength for each of the individual recommendations, and agreement among experts was excellent. Prominent features of these recommendations are targeting remission, preventing damage, and improving health-related quality of life. It is anticipated that 'treating-to-target' can and will be applicable to the care of patients with SLE in the future.

\section{Author affiliations}

${ }^{1}$ Department of Medicine, Unit for Clinical Therapy Research, Inflammatory Diseases, Stockholm, Sweden, Karolinska Institutet, Stockholm, Sweden

${ }^{2}$ Rheumatology Unit, University of Pisa, Pisa, Italy

${ }^{3}$ Department of Internal Medicine, University of Crete, Heraklion, Greece

${ }^{4}$ Division of Medicine, UCL, Centre for Rheumatology Research, London, UK

${ }^{5}$ Department of Dermatology, University of Muenster, Muenster, Germany

${ }^{6}$ Department of Dermatology, Lupus Europe, Romford, UK

${ }^{7}$ Medical Faculty, Department of Internal Medicine III, Technical University of Dresden, Dresden, Germany

${ }^{8}$ Department of Rheumatology and Clinical Immunology, University Medical Center Groningen, University of Groningen, Groningen, The Netherlands

${ }^{9}$ Department of Medicne/Rheumatology-Clinical Immunology, University of Crete, Heraklion, Greece

${ }^{10}$ Arc Epidemiology Unit, University of Manchester, Manchester, UK

${ }^{11}$ Department of Autoimmune Diseases, Hospital Clinic, Barcelona, Spain

${ }^{12}$ Division of Clinical Epidemiology, McGill University, Montreal, Canada

${ }^{13}$ Service de Médicine Interne 2, Hôpital Pitié-Salpêtrière, Paris, France

${ }^{14}$ Department of Rheumatology and Immunology, University of Pecs, Pecs, Hungary

${ }^{15}$ Department of Rheumatology and Clinical Immunology, University Medical Center

Utrecht, The Netherlands, Utrecht, The Netherlands

${ }^{16}$ Department of Medicne/Rheumatology and Clinical Immunology, Charite University Hospital, Berlin, Germany

${ }^{17}$ Rheumatology Research Group, Birmingham University Medical School,

Birmingham, West Midlands, UK

${ }^{18}$ Department of Internal Medicine, Medical University of Graz, Graz, Austria

${ }^{19}$ Department of Rheumatology, Université Catholique de Louvain, Bruxelles,

Belgium

${ }^{20}$ Department of Internal Medicine, Istanbul University, Istanbul, Turkey

${ }^{21}$ Department of Rheumatology—4242, Rigshospitalet, Copenhagen University

Hospital, Copenhagen, Denmark

${ }^{22}$ Department of Medicine, Addenbrooke's Hospital, Cambridge, UK

${ }^{23}$ Institute of Rheumatology, Warsaw, Poland

${ }^{24}$ Discipline of Rheumatology, Universidade do Estado do Rio de Janeiro, Rio de

Janeiro, Brazil

${ }^{25}$ Assistance Publique-Hôpitaux de Paris, Hôpitaux Universitaires Paris Sud, Le Kremlin-Bicêtre, France

${ }^{26}$ Southern Clinical School, Monash University, Clayton, Victoria, Australia

${ }^{27}$ Rheumatology Department, University of Santo Tomas Hospital, Manila,

Philippines

${ }^{28}$ Med. Abteilung für Nephrologie und Dialyse, Wilhelminenspital, Wien, Austria

${ }^{29}$ Department of Rheumatology, University College London, London, UK

${ }^{30}$ National Institute for Rheumatic Diseases, Piestany, Slovakia

${ }^{31}$ Department of Rheumatology, Medical University of Vienna, Vienna, Austria

${ }^{32}$ Unidade Imunologia Clínica, Department of Medicine, Hospital Santo António,

Porto, Portugal

${ }^{33}$ Department of Rheumatology, VU University Medical Center, Amsterdam, The Netherlands

${ }^{34}$ Department of Rheumatology, Odense University Hospital, Odense, Denmark

${ }^{35}$ Department of Nephrology, Moscow State Medicine and Dentistry University,

Moscow, Russian Federation

${ }^{36}$ Lanarkshire Centre for, East Kilbride, UK

${ }^{37}$ Department of Rheumatology, Heinrich-Heine-University Duesseldorf, Duesseldorf, Germany

Funding Supported by unrestricted grants from GSK and UCB pharmas. 
Competing interests RvV reports grants from GSK, UCB, during the conduct of the study; grants and personal fees from AbbVie, Biotest, BMS, GSK, Janssen, Lilly, Merck, Pfizer, Roche, UCB, Vertex, outside the submitted work. MM, DI, HB, DB, JS, AV: disclosures not reported. GB reports personal fees from GSK, UCB, outside the submitted work. IB reports support from The Manchester Academic health Science Centre, the NIHR Biomedical Research Unit Funding Scheme, the NIHR Manchester Wellcome Trust Clinical Research Facility and the NIHR Manchester Biomedical Research Centre. RC reports collaborations as Consultant/Advisor for GlaxoSmithKline, Human Genome Sciences, Roche, Medimmune, UCB, Cephalon, Lilly, Inova, Werfen group and Minarini Diagnostics. AK, LC, NC-C, RD, WG, SJ, MI, AJ-G, AL, IN, AR, JR, HZ, AZ: no disclosures to report. KL reports grants from LUPUS EUROPE, a UK based Charity which has received restricted and unrestricted funds for operation and projects from GSK, UCB, Lilly, Roche, Merck and other non-pharmaceutical companies, outside the submitted work. MA reports grants and personal fees from AbbVie, Chugai, GSK, MSD, Novartis, Pfizer, Roche, Sandoz, $U C B$, outside the submitted work. AC reports personal fees from GSK, Lilly, BMS, outside the submitted work. FH reports grants and personal fees from AbbVie, Roche, GSK, Merck, Pfizer, Lilly, Neovacs, UCB, outside the submitted work. TD reports personal fees from Lilly, Roche/Chugai, Sanofi, Takeda, UCB, outside the submitted work. CG reports personal fees from Amgen, BMS, GSK, Medlmmune, Merck-Serono, UCB, outside the submitted work. DJ reports grants from Roche, Genentech, personal fees from GSK, Roche, TEVA, other from GSK, Roche, outside the submitted work. RL reports grants and personal fees from AbbVie, Anthera, AstraZeneca, GSK, INOVA, Janssen, Pfizer, Roche, outside the submitted work. XM reports grants and personal fees from BMS, GSK, Pfizer, Roche, Sanofi, UCB, outside the submitted work. EM reports grants and personal fees from AbbVie, AstraZeneca, GSK, Lilly, Pfizer, Roche, UCB, outside the submitted work. SN has received honoraria from GSK, Roche, Pfizer. CV has received fees from advisory boards and conferences from Roche, BMS, Gilead, Janssen, MSD, Abbott, Pfizer, Boehringer Ingelheim, Aspreva and GSK. AV reports personal fees from GSK, outside the submitted work. MS reports grants and personal fees from AbbVie, AstraZeneca, GSK, Lilly, Pfizer, Roche, UCB, outside the submitted work.

Provenance and peer review Not commissioned; externally peer reviewed.

\section{REFERENCES}

1 Swales JD. Pharmacological treatment of hypertension. Lancet 1994;344:380-5.

2 Eastman RC, Keen $\mathrm{H}$. The impact of cardiovascular disease on people with diabetes: the potential for prevention. Lancet 1997;350(Suppl 1):SI29-32.

3 Grigor C, Capell H, Stirling A, et al. Effect of a treatment strategy of tight control for rheumatoid arthritis (the TICORA study): a single-blind randomised controlled trial. Lancet 2004;364:263-9.

4 Verstappen SM, Jacobs JW, van der Veen MJ, et al. Intensive treatment with methotrexate in early rheumatoid arthritis: aiming for remission. Computer Assisted Management in Early Rheumatoid Arthritis (CAMERA, an open-label strategy trial). Ann Rheum Dis 2007;66:1443-9.

5 Mottonen T, Hannonen P, Leirisalo-Repo M, et al. Comparison of combination therapy with single-drug therapy in early rheumatoid arthritis: a randomised trial. FIN-RACo trial group. Lancet 1999;353:1568-73.

6 Smolen JS, Aletaha D, Bijlsma JW, et al. Treating rheumatoid arthritis to target: recommendations of an international task force. Ann Rheum Dis 2010;69:631-7.

7 Smolen JS, Braun J, Dougados M, et al. Treating spondyloarthritis, including ankylosing spondylitis and psoriatic arthritis, to target: recommendations of an international task force. Ann Rheum Dis 2014;73:6-16.

8 Nived 0 , Jonsen A, Bengtsson AA, et al. High predictive value of the Systemic Lupus International Collaborating Clinics/American College of Rheumatology damage index for survival in systemic lupus erythematosus. I Rheumatol 2002;29:1398-400.

9 AGREE Collaboration. Development and validation of an international appraisal instrument for assessing the quality of clinical practice guidelines: the AGREE project. Qual Saf Health Care 2003;12:18-23.

10 Group OLoEW. OCEBM Levels of Evidence Working Group. The Oxford Levels of Evidence 2. Oxford Centre for Evidence-Based Medicine. http://www.cebm.net/ index.aspx?0=5653. In: Group OLoEW, ed., 2011.

11 Mosca M, Tani C, Aringer M, et al. European League Against Rheumatism recommendations for monitoring patients with systemic lupus erythematosus in clinical practice and in observational studies. Ann Rheum Dis 2010;69:1269-74.

12 Gladman D, Ginzler E, Goldsmith C, et al. The development and initial validation of the Systemic Lupus International Collaborating Clinics/American College of Rheumatology damage index for systemic lupus erythematosus. Arthritis Rheum 1996:39:363-9

13 Petri M, Purvey $\mathrm{S}$, Fang $\mathrm{H}$, et al. Predictors of organ damage in systemic lupus erythematosus: The Hopkins Lupus Cohort. Arthritis Rheum 2012;64:4021-8.

14 Ibanez D, Urowitz MB, Gladman DD. Summarizing disease features over time: I. Adjusted mean SLEDAI derivation and application to an index of disease activity in lupus. J Rheumatol 2003:30:1977-82.
15 Andrade RM, Alarcon GS, Fernandez M, et al. Accelerated damage accrual among men with systemic lupus erythematosus: XLIV. Results from a multiethnic US cohort. Arthritis Rheum 2007:56:622-30.

16 Lopez R, Davidson JE, Beeby MD, et al. Lupus disease activity and the risk of subsequent organ damage and mortality in a large lupus cohort. Rheumatology (Oxford) 2012;51:491-8.

17 Bertoli AM, Vila LM, Apte M, et al. Systemic lupus erythematosus in a multiethnic US cohort LUMINA LI: anaemia as a predictor of disease activity and damage accrual. Rheumatology (Oxford) 2007;46:1471-6.

18 Stoll T, Sutcliffe N, Mach J, et al. Analysis of the relationship between disease activity and damage in patients with systemic lupus erythematosus - a 5-yr prospective study. Rheumatology (Oxford) 2004;43:1039-44.

19 Andrade RM, Alarcon GS, Gonzalez LA, et al. Seizures in patients with systemic lupus erythematosus: data from LUMINA, a multiethnic cohort (LUMINA LIV). Ann Rheum Dis 2008;67:829-34.

20 Manger K, Manger B, Repp R, et al. Definition of risk factors for death, end stage renal disease, and thromboembolic events in a monocentric cohort of 338 patients with systemic lupus erythematosus. Ann Rheum Dis 2002;61:1065-70.

21 Arends S, Grootscholten C, Derksen RH, et al. Long-term follow-up of a randomised controlled trial of azathioprine/methylprednisolone versus cyclophosphamide in patients with proliferative lupus nephritis. Ann Rheum Dis 2012;71:966-73.

22 Reich HN, Gladman DD, Urowitz MB, et al. Persistent proteinuria and dyslipidemia increase the risk of progressive chronic kidney disease in lupus erythematosus. Kidney Int 2011;79:914-20.

23 Tisseverasinghe A, Lim S, Greenwood C, et al. Association between serum total cholesterol level and renal outcome in systemic lupus erythematosus. Arthritis Rheum 2006;54:2211-19.

24 Drenkard C, Villa AR, Garcia-Padilla C, et al. Remission of systematic lupus erythematosus. Medicine 1996;75:88-98.

25 Nossent J, Kiss E, Rozman B, et al. Disease activity and damage accrual during the early disease course in a multinational inception cohort of patients with systemic lupus erythematosus. Lupus 2010;19:949-56.

26 Illei GG, Takada K, Parkin D, et al. Renal flares are common in patients with severe proliferative lupus nephritis treated with pulse immunosuppressive therapy: long-term followup of a cohort of 145 patients participating in randomized controlled studies. Arthritis Rheum 2002;46:995-1002.

27 Mok CC, Ying KY, Tang S, et al. Predictors and outcome of renal flares after successful cyclophosphamide treatment for diffuse proliferative lupus glomerulonephritis. Arthritis Rheum 2004;50:2559-68.

28 Moroni G, Quaglini S, Gravellone L, et al. Membranous nephropathy in systemic lupus erythematosus: long-term outcome and prognostic factors of 103 patients. Semin Arthritis Rheum 2012:41:642-51.

29 Morand E, Franklyn K, Lau CS, et al. Consensus Definition and Preliminary Validation of a Low Disease Activity State in Systemic Lupus Erythematosus. Poster, ACR Annual Congress, 2013.

30 Strand V, Gladman D, Isenberg $D$, et al. Outcome measures to be used in clinical trials in systemic lupus erythematosus. J Rheumatol 1999;26:490-7.

31 Furie $\mathrm{R}$, Petri $\mathrm{M}$, Zamani $\mathrm{O}$, et al. A phase III, randomized, placebo-controlled study of belimumab, a monoclonal antibody that inhibits B lymphocyte stimulator, in patients with systemic lupus erythematosus. Arthritis Rheum 2011;63:3918-30.

32 Griffiths B, Emery P, Ryan V, et al. The BILAG multi-centre open randomized controlled trial comparing ciclosporin vs azathioprine in patients with severe SLE. Rheumatology (Oxford) 2010;49:723-32.

33 Merrill J, Buyon J, Furie R, et al. Assessment of flares in lupus patients enrolled in a phase II/III study of rituximab (EXPLORER). Lupus 2011;20:709-16.

34 Navarra SV, Guzman RM, Gallacher AE, et al. Efficacy and safety of belimumab in patients with active systemic lupus erythematosus: a randomised, placebo-controlled, phase 3 trial. Lancet 2011;377:721-31.

35 Dooley MA, Jayne $D$, Ginzler EM, et al. Mycophenolate versus azathioprine as maintenance therapy for lupus nephritis. N Engl I Med 2011;365:1886-95.

36 Houssiau FA, D'Cruz D, Sangle S, et al. Azathioprine versus mycophenolate mofetil for long-term immunosuppression in lupus nephritis: results from the MAINTAIN Nephritis Trial. Ann Rheum Dis 2010;69:2083-9.

37 Moroni G, Quaglini S, Gallelli B, et al. The long-term outcome of 93 patients with proliferative lupus nephritis. Nephrol Dial Transplant 2007;22:2531-9.

38 Mosca M, Bencivelli W, Neri R, et al. Renal flares in 91 SLE patients with diffuse proliferative glomerulonephritis. Kidney Int 2002;61:1502-9.

39 Urowitz $\mathrm{MB}$, Feletar $\mathrm{M}$, Bruce $\mathrm{IN}$, et al. Prolonged remission in systemic lupus erythematosus. J Rheumatol 2005;32:1467-72.

40 Birnbaum J, Petri $\mathrm{M}$, Thompson $\mathrm{R}$, et al. Distinct subtypes of myelitis in systemic lupus erythematosus. Arthritis Rheum 2009:60:3378-87.

41 Swaak AJ, Nossent JC, Bronsveld W, et al. Systemic lupus erythematosus. I. Outcome and survival: Dutch experience with 110 patients studied prospectively. Ann Rheum Dis 1989;48:447-54.

42 Bandeira M, Buratti S, Bartoli M, et al. Relationship between damage accrual, disease flares and cumulative drug therapies in juvenile-onset systemic lupus erythematosus. Lupus 2006;15:515-20. 
43 Laustrup $H$, Voss A, Green A, et al. SLE disease patterns in a Danish population-based lupus cohort: an 8-year prospective study. Lupus 2010;19:239-46.

44 Mok CC, Ho CT, Wong RW, et al. Damage accrual in southern Chinese patients with systemic lupus erythematosus. J Rheumatol 2003:30:1513-19.

45 Ho A, Barr SG, Magder LS, et al. A decrease in complement is associated with increased renal and hematologic activity in patients with systemic lupus erythematosus. Arthritis Rheum 2001;44:2350-7

46 Ho A, Magder LS, Barr SG, et al. Decreases in anti-double-stranded DNA levels are associated with concurrent flares in patients with systemic lupus erythematosus. Arthritis Rheum 2001;44:2342-9.

47 Mirzayan MJ, Schmidt RE, Witte T. Prognostic parameters for flare in systemic lupus erythematosus. Rheumatology (Oxford) 2000;39:1316-19.

48 Petri $M$, Singh $S$, Tesfasyone $H$, et al. Prevalence of flare and influence of demographic and serologic factors on flare risk in systemic lupus erythematosus: a prospective study. J Rheumatol 2009:36:2476-80.

49 Swaak AJ, Groenwold J, Bronsveld W. Predictive value of complement profiles and anti-dsDNA in systemic lupus erythematosus. Ann Rheum Dis 1986:45:359-66.

50 ter Borg EJ, Horst G, Hummel EJ, et al. Measurement of increases in anti-double-stranded DNA antibody levels as a predictor of disease exacerbation in systemic lupus erythematosus. A long-term, prospective study. Arthritis Rheum 1990;33:634-43.

$51 \mathrm{Ng} \mathrm{KP}$, Manson JJ, Rahman A, et al. Association of antinucleosome antibodies with disease flare in serologically active clinically quiescent patients with systemic lupus erythematosus. Arthritis Rheum 2006:55:900-4.

52 Bijl M, Dijstelbloem HM, Oost WW, et al. IgG subclass distribution of autoantibodies differs between renal and extra-renal relapses in patients with systemic lupus erythematosus. Rheumatology (Oxford) 2002;41:62-7.

53 Birmingham DJ, Irshaid F, Nagaraja HN, et al. The complex nature of serum C3 and C4 as biomarkers of lupus renal flare. Lupus 2010;19:1272-80.

54 Bootsma H, Spronk P, Derksen R, et al. Prevention of relapses in systemic lupus erythematosus. Lancet 1995:345:1595-9.

55 Tseng CE, Buyon JP, Kim M, et al. The effect of moderate-dose corticosteroids in preventing severe flares in patients with serologically active, but clinically stable, systemic lupus erythematosus: findings of a prospective, randomized, double-blind, placebo-controlled trial. Arthritis Rheum 2006:54:3623-32.

56 Steiman AJ, Gladman DD, Ibanez D, et al. Prolonged serologically active clinically quiescent systemic lupus erythematosus: frequency and outcome. J Rheumatol 2010;37:1822-7.

57 Steiman AJ, Gladman DD, Ibanez D, et al. Outcomes in patients with systemic lupus erythematosus with and without a prolonged serologically active clinically quiescent period. Arthritis Care Res 2012;64:511-18.

58 Chambers SA, Allen E, Rahman A, et al. Damage and mortality in a group of British patients with systemic lupus erythematosus followed up for over 10 years. Rheumatology (Oxford) 2009:48:673-5.

59 Hanly JG, Urowitz MB, Su L, et al. Seizure disorders in systemic lupus erythematosus results from an international, prospective, inception cohort study. Ann Rheum Dis 2012;71:1502-9.

60 Mak A, Cheung MW, Chiew HJ, et al. Global trend of survival and damage of systemic lupus erythematosus: meta-analysis and meta-regression of observational studies from the 1950s to 2000s. Semin Arthritis Rheum 2012;41:830-9.

61 Ramsey-Goldman R, Alarcon GS, McGwin G, et al. Time to seizure occurrence and damage in PROFILE, a multi-ethnic systemic lupus erythematosus cohort. Lupus 2008:17:177-84

62 Dua AB, Touma Z, Toloza S, et al. Top 10 recent developments in health-related quality of life in patients with systemic lupus erythematosus. Curr Rheumatol Rep 2013;15:380.

63 Kiani AN, Petri M. Quality-of-life measurements versus disease activity in systemic lupus erythematosus. Curr Rheumatol Rep 2010;12:250-8.

64 Jolly M, Pickard AS, Wilke C, et al. Lupus-specific health outcome measure for US patients: the LupusQoL-US version. Ann Rheum Dis 2010:69:29-33.

65 McElhone K, Castelino M, Abbott J, et al. The LupusQoL and associations with demographics and clinical measurements in patients with systemic lupus erythematosus. J Rheumatol 2010;37:2273-9.

66 Zhu TY, Tam LS, Lee VW, et al. Relationship between flare and health-related quality of life in patients with systemic lupus erythematosus. J Rheumatol 2010;37:568-73.

67 Aggarwal R, Wilke CT, Pickard AS, et al. Psychometric properties of the EuroQol-5D and Short Form-6D in patients with systemic lupus erythematosus. J Rheumatol 2009;36:1209-16.

68 Doria A, Rinaldi S, Ermani M, et al. Health-related quality of life in Italian patients with systemic lupus erythematosus. II. Role of clinical, immunological and psychological determinants. Rheumatology (Oxford) 2004:43:1580-6.

69 Yazdany J, Trupin L, Gansky SA, et al. Brief index of lupus damage: a patient-reported measure of damage in systemic lupus erythematosus. Arthritis Care Res 2011;63:1170-7.

70 Appenzeller $\mathrm{S}$, Clarke $\mathrm{AE}$, Panopalis $\mathrm{P}$, et al. The relationship between renal activity and quality of life in systemic lupus erythematosus. J Rheumatol 2009:36:947-52.
71 Kuriya B, Gladman DD, Ibanez D, et al. Quality of life over time in patients with systemic lupus erythematosus. Arthritis Rheum 2008;59:181-5.

72 Mok CC, Ho LY, Cheung MY, et al. Effect of disease activity and damage on quality of life in patients with systemic lupus erythematosus: a 2-year prospective study. Scand J Rheumatol 2009;38:121-7.

73 Moorthy LN, Peterson MG, Hassett AL, et al. Relationship between health-related quality of life and SLE activity and damage in children over time. Lupus 2009:18:622-9.

74 Grootscholten C, Snoek FJ, Bijl M, et al. Health-related quality of life and treatment burden in patients with proliferative lupus nephritis treated with cyclophosphamide or azathioprine/methylprednisolone in a randomized controlled trial. J Rheumatol 2007;34:1699-707.

75 Strand V, Aranow C, Cardiel MH, et al. Improvement in health-related quality of life in systemic lupus erythematosus patients enrolled in a randomized clinical trial comparing LJP 394 treatment with placebo. Lupus 2003;12:677-86.

76 van Vollenhoven RF, Petri MA, Cervera $R$, et al. Belimumab in the treatment of systemic lupus erythematosus: high disease activity predictors of response. Ann Rheum Dis 2012;71:1343-9.

77 So MW, Koo BS, Kim YG, et al. Predictive value of remission status after 6 months induction therapy in patients with proliferative lupus nephritis: a retrospective analysis. Clin Rheumatol 2011;30:1399-405.

78 Faurschou M, Dreyer L, Kamper AL, et al. Long-term mortality and renal outcome in a cohort of 100 patients with lupus nephritis. Arthritis Care Res 2010;62:873-80.

79 Dall'era $M$, Stone $D$, Levesque $V$, et al. Identification of biomarkers that predict response to treatment of lupus nephritis with mycophenolate mofetil or pulse cyclophosphamide. Arthritis Care Res 2011;63:351-7.

80 Esdaile JM, Levinton C, Federgreen W, et al. The clinical and renal biopsy predictors of long-term outcome in lupus nephritis: a study of 87 patients and review of the literature. Q J Med 1989:72:779-833.

81 Ioannidis JP, Boki KA, Katsorida ME, et al. Remission, relapse, and re-remission of proliferative lupus nephritis treated with cyclophosphamide. Kidney Int 2000;57:258-64.

82 Jacobsen S, Starklint H, Petersen J, et al. Prognostic value of renal biopsy and clinical variables in patients with lupus nephritis and normal serum creatinine. Scand J Rheumatol 1999:28:288-99.

83 Rzany B, Coresh J, Whelton PK, et al. Risk factors for hypercreatinemia in patients with systemic lupus erythematosus. Lupus 1999;8:532-40.

84 Harisdangkul V, Doorenbos D, Subramony SH. Lupus transverse myelopathy: better outcome with early recognition and aggressive high-dose intravenous corticosteroid pulse treatment. J Neurol 1995;242:326-31.

85 Lu X, Gu Y, Wang Y, et al. Prognostic factors of lupus myelopathy. Lupus 2008:17:323-8

86 Fernandes Moca Trevisani V, Castro AA, Ferreira Neves Neto J, et al. Cyclophosphamide versus methylprednisolone for treating neuropsychiatric involvement in systemic lupus erythematosus. Cochrane Database Syst Rev 2013;2 CD002265.

87 Henderson L, Masson P, Craig JC, et al. Treatment for lupus nephritis. Cochrane Database Syst Rev 2012;12:CD002922.

88 Boumpas DT, Austin HA III, Vaughn EM, et al. Controlled trial of pulse methylprednisolone versus two regimens of pulse cyclophosphamide in severe lupus nephritis. Lancet 1992:340:741-5.

89 Ginzler E, Sharon E, Diamond H, et al. Long-term maintenance therapy with azathioprine in systemic lupus erythematosus. Arthritis Rheum 1975; 18:27-34

90 Mok CC, Ying KY, Ng WL, et al. Long-term outcome of diffuse proliferative lupus glomerulonephritis treated with cyclophosphamide. Am J Med 2006;119:355 e25-33.

91 Houssiau FA, Vasconcelos C, D'Cruz D, et al. The 10-year follow-up data of the Euro-Lupus Nephritis Trial comparing low-dose and high-dose intravenous cyclophosphamide. Ann Rheum Dis 2010;69:61-4

92 Laskari K, Tzioufas AG, Antoniou A, et al. Longterm followup after tapering mycophenolate mofetil during maintenance treatment for proliferative lupus nephritis. J Rheumatol 2011;38:1304-8.

93 Yap DY, Ma MK, Mok MM, et al. Long-term data on corticosteroids and mycophenolate mofetil treatment in lupus nephritis. Rheumatology (Oxford) 2013;52:480-6.

94 Thamer M, Hernan MA, Zhang Y, et al. Prednisone, lupus activity, and permanent organ damage. J Rheumatol 2009;36:560-4.

95 Rygg M, Pistorio A, Ravelli A, et al. A longitudinal PRINTO study on growth and puberty in juvenile systemic lupus erythematosus. Ann Rheum Dis 2012:71:511-17.

96 Mazzantini M, Torre C, Miccoli M, et al. Adverse events during longterm low-dose glucocorticoid treatment of polymyalgia rheumatica: a retrospective study. J Rheumatol 2012;39:552-7.

97 Mazzantini M, Talarico R, Doveri M, et al. Incident comorbidity among patients with rheumatoid arthritis treated or not with low-dose glucocorticoids: a retrospective study. J Rheumatol 2010;37:2232-6. 
98 Ruiz-Irastorza G, Equrbide MV, Martinez-Berriotxoa A, et al. Antiphospholipid antibodies predict early damage in patients with systemic lupus erythematosus. Lupus 2004;13:900-5.

99 Mok MY, Chan EY, Fong DY, et al. Antiphospholipid antibody profiles and their clinical associations in Chinese patients with systemic lupus erythematosus. J Rheumatol 2005;32:622-8.

100 Tarr T, Lakos G, Bhattoa HP, et al. Analysis of risk factors for the development of thrombotic complications in antiphospholipid antibody positive lupus patients. Lupus 2007;16:39-45.

101 Ruiz-Irastorza G, Crowther M, Branch W, et al. Antiphospholipid syndrome. Lancet 2010;376:1498-509.

102 Tektonidou MG, Laskari K, Panagiotakos DB, et al. Risk factors for thrombosis and primary thrombosis prevention in patients with systemic lupus erythematosus with or without antiphospholipid antibodies. Arthritis Rheum 2009;61:29-36

103 Crowther MA, Ginsberg JS, Julian J, et al. A comparison of two intensities of warfarin for the prevention of recurrent thrombosis in patients with the antiphospholipid antibody syndrome. N Engl J Med 2003;349:1133-8.

104 Finazzi G, Marchioli R, Brancaccio V, et al. A randomized clinical trial of high-intensity warfarin vs. conventional antithrombotic therapy for the prevention of recurrent thrombosis in patients with the antiphospholipid syndrome (WAPS). J Thromb Haemost 2005;3:848-53.

105 Khamashta MA, Cuadrado MJ, Mujic F, et al. The management of thrombosis in the antiphospholipid-antibody syndrome. N Engl J Med 1995;332:993-7.

106 Tan BE, Thong BY, Shivananda S, et al. Clinical manifestations and outcomes of antithrombotic treatment of the Tan Tock Seng Hospital Singapore antiphospholipid syndrome cohort. Lupus 2009;18:752-8.

107 Ruiz-Irastorza G, Ramos-Casals M, Brito-Zeron P, et al. Clinical efficacy and side effects of antimalarials in systemic lupus erythematosus: a systematic review. Ann Rheum Dis 2010;69:20-8.

108 Tsakonas E, Joseph L, Esdaile JM, et al. A long-term study of hydroxychloroquine withdrawal on exacerbations in systemic lupus erythematosus. The Canadian Hydroxychloroquine Study Group. Lupus 1998;7:80-5.

109 Bezerra EL, Vilar MJ, da Trindade Neto PB, et al. Double-blind, randomized, controlled clinical trial of clofazimine compared with chloroquine in patients with systemic lupus erythematosus. Arthritis Rheum 2005;52:3073-8.
110 Cavazzana I, Sala R, Bazzani C, et al. Treatment of lupus skin involvement with quinacrine and hydroxychloroquine. Lupus 2009;18:735-9.

111 Fessler BJ, Alarcon GS, McGwin G Jr, et al. Systemic lupus erythematosus in three ethnic groups: XVI. Association of hydroxychloroquine use with reduced risk of damage accrual. Arthritis Rheum 2005;52:1473-80.

112 Ibanez D, Gladman DD, Urowitz MB. Adjusted mean Systemic Lupus Erythematosus Disease Activity Index-2K is a predictor of outcome in SLE. J Rheumatol 2005;32:824-7.

113 Ruiz-Irastorza G, Egurbide MV, Pijoan Jl, et al. Effect of antimalarials on thrombosis and survival in patients with systemic lupus erythematosus. Lupus 2006;15:577-83.

114 Urowitz MB, Gladman DD, Tom BD, et al. Changing patterns in mortality and disease outcomes for patients with systemic lupus erythematosus. J Rheumatol 2008;35:2152-8.

115 Zheng ZH, Zhang LJ, Liu WX, et al. Predictors of survival in Chinese patients with lupus nephritis. Lupus 2012;21:1049-56.

116 Duran-Barragan S, McGwin G Jr, Vila LM, et al. cohort LamU. Angiotensinconverting enzyme inhibitors delay the occurrence of renal involvement and are associated with a decreased risk of disease activity in patients with systemic lupus erythematosus - results from LUMINA (LIX): a multiethnic US cohort. Rheumatology (Oxford) 2008:47:1093-6.

117 McLaurin EY, Holliday SL, Williams P, et al. Predictors of cognitive dysfunction in patients with systemic lupus erythematosus. Neurology 2005;64:297-303.

118 Burgos PI, Vila LM, Reveille JD, et al. Peripheral vascular damage in systemic lupus erythematosus: data from LUMINA, a large multi-ethnic U.S. cohort (LXIX). Lupus 2009:18:1303-8.

119 Norby GE, Holme I, Fellstrom B, et al. Effect of fluvastatin on cardiac outcomes in kidney transplant patients with systemic lupus erythematosus: a randomized placebo-controlled study. Arthritis Rheum 2009;60:1060-4.

120 Petri MA, Kiani AN, Post W, et al. Lupus Atherosclerosis Prevention Study (LAPS). Ann Rheum Dis 2011;70:760-5.

121 Jonsdottir T, Gunnarsson I, Risselada A, et al. Treatment of refractory SLE with rituximab plus cyclophosphamide: clinical effects, serological changes, and predictors of response. Ann Rheum Dis 2008;67:330-4.

122 Terrier B, Amoura Z, Ravaud $\mathrm{P}$, et al. Safety and efficacy of rituximab in systemic lupus erythematosus: results from 136 patients from the French Autolmmunity and Rituximab registry. Arthritis Rheum 2010;62:2458-66. 